\title{
The vacuum bell for conservative treatment of pectus excavatum: the Basle experience
}

\author{
Frank-Martin Haecker
}

Published online: 15 January 2011

(C) Springer-Verlag 2011

\begin{abstract}
Objective Surgical repair of pectus excavatum $(\mathrm{PE})$ in childhood is a well-established procedure. Previously used operative techniques to correct PE were largely based on the Ravitch technique. Since about 10 years, the minimally invasive repair (MIRPE) by Nuss is well established. Conservative treatment with the vacuum bell to elevate the funnel in patients with $\mathrm{PE}$ represents a potential alternative to surgery in selected patients.

Methods A suction cup is used to create a vacuum at the anterior chest wall. Three different sizes of vacuum bell exist which are selected according to the individual patients age. When creating the vacuum, the lift of the sternum is obvious and remains for a different time period. The device should be used for a minimum of $30 \mathrm{~min}$ (twice/day), and may be used up to a maximum of several hours daily.

Results One hundred and thirty-three patients (110 males, 23 females) aged from 3 to 61 years (median 16.21 years) used the vacuum bell for 1 to a maximum of 36 months. Computed tomographic scans showed that the device lifted the sternum and ribs immediately. In addition, this was confirmed thoracoscopically during the MIRPE procedure. One hundred and five patients showed a permanent lift of the sternum for more than $1 \mathrm{~cm}$ after 3 months of daily application. Thirteen patients stopped the application and underwent MIRPE. Relevant side effects were not noted.

Conclusion The vacuum bell has proved to be an alternative therapeutic option in selected patients suffering from PE. The initial results proved to be dramatic, but long-term
\end{abstract}

F.-M. Haecker $(\bowtie)$

Department of Pediatric Surgery, University Children's Hospital, Spitalstrasse 33, 4056 Basel, Switzerland

e-mail: frankmartin.haecker@ukbb.ch results are so far lacking, and further evaluation and follow-up studies are necessary.

Keywords Pectus excavatum - Conservative treatment . Vacuum bell

\section{Introduction}

Pectus excavatum (PE) is the most common chest wall malformation and one of the most frequent major congenital anomalies, occurring in approximately one in every 300 births [1]. PE can be repaired by several approaches. The technique described by Ravitch [2] and modifications require exposure of the cartilages/sternal junctions, removal of abnormal cartilages, and fixation of the sternum in a more normal position. In 1998, a new technique of minimally invasive repair of PE (MIRPE) was first described by Nuss [3] to avoid several operative features of the modified Ravitch repair procedure. Today, the MIRPE technique is well established and represents a common used technique [4-9]. The shorter operating time, smaller incisions, and considerably less dissection have made the MIRPE procedure very appealing both to surgeons and patients, thereby resulting in a large increase of the number of patients requesting operative treatment, and consequently, an increase of the number of PE repairs. However, with the widespread use of the MIRPE procedure, the character and number of complications have increased [4, 6-8, 10-12]. Above all, recent studies report on an increasing number of near fatal complications [12-18]. Additionally, in many cases of PE, the degree of pectus deformity does not immediately warrant surgery. Some patients are reluctant to undergo surgery because of the pain associated with postoperative recovery and the 
risk of imperfect results. In this situation, the introduction of the vacuum bell for conservative treatment of PE has made this alternative therapy a focus of interest of patients.

The procedure of applying a vacuum to elevate the sternum was first used more than 100 years ago [19]. Despite the risks and unsatisfactory results after operative therapy for some patients, there has been little progress in the therapeutic use of the vacuum therapy during the last few decades. In the meantime, materials have improved and the vacuum devices can now exert strong forces. Our initial results using this method proved to be promising $[20,21]$. Today, we report our ongoing experience using the vacuum bell for conservative treatment of PE. Note that a subset of these patients was reported previously [20, 21].

\section{The vacuum bell}

A suction cup is used to create a vacuum at the chest wall. The technique in detail is described previously [20]. Three different sizes exist allowing selection according to the individual patient's age (Fig. 1a). The medium size model is available in a supplemental version with a reinforced silicon wall (type "bodybuilder"), e.g., for adult patients with a small deep PE. Additionally, a model fitted for young girls and women is available (Fig. 1b). Pilot studies performed by Schier and Bahr [22] showed that the device lifted the sternum and ribs immediately. We could also confirm this effect by thoracoscopy during the MIRPE procedure. We recommend using the vacuum bell for a minimum of $30 \mathrm{~min}$, twice per day. The device may be used up to a maximum of several hours daily.
Fig. 1 a Vacuum bell in three different sizes (left $16 \mathrm{~cm}$, middle $19 \mathrm{~cm}$, and right $26 \mathrm{~cm}$ in diameter). b Vacuum bell, model for adolescent girls/ women
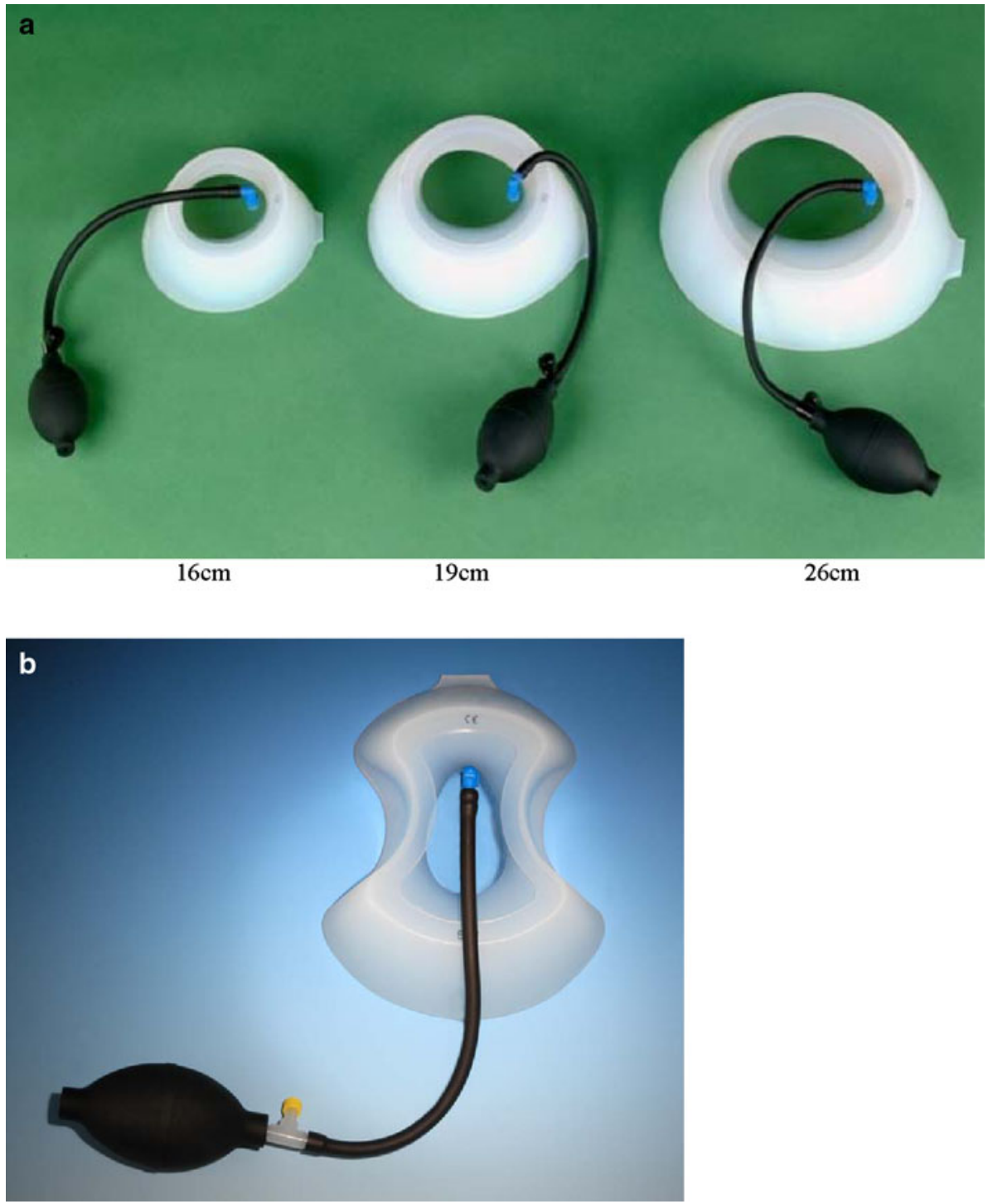
Indication for conservative therapy with the vacuum bell includes patients who present with mild degree of PE, who want to avoid surgical procedure, and who are reluctant to undergo surgery because of pain associated with the operation. Additionally, patients' concerns about imperfect results after surgery have to be noticed. Contraindications of the method comprise skeletal disorders, vasculopathies, coagulopathies and cardiac disorders [20]. To exclude these disorders, a standardized evaluation protocol was routinely performed before beginning the therapy. Complications and relevant side effects include subcutaneous hematoma, petechial bleeding, dorsalgia, and transient paresthesia of the upper extremities during the application.

\section{Patients and methods}

One hundred and thirty-three patients (110 males, 23 females) aged from 3 to 61 years (median 16.21 years) were treated with the vacuum bell for 1 to a maximum of 36 months. Standardized evaluation before starting the procedure included history of the patient and his family, clinical examination, cardiac evaluation with electrocardiogram, and echocardiography and photo documentation. The depth of PE was measured in a standardized supine position. Patients underwent follow-up at 3-6 months intervals including clinical examination and measurement of depth of PE.

The first application of the vacuum bell occurred under supervision of the attending doctor. The length of time of daily application of the vacuum bell varied widely between patients. Some patients followed the user instructions and applied the device twice daily for $30 \mathrm{~min}$ each. Patients under the age of 10 years used the device under supervision of their parents or caregivers. Some of the adult patients used the vacuum bell up to $8 \mathrm{~h}$ daily during office hours. Adolescent boys applied the device every night for 7-8 h. In fact, the duration and frequency of daily application depends on the patients' individual decision and motivation.

Since 2006, in our institution, the vacuum bell was used routinely during the MIRPE procedure to elevate the sternum to allow a safer passage of the Loren ${ }^{\circledR}$ introducer as well as the pectus bar.

\section{Results}

During the first 1-5 applications, most of the patients experienced moderate pain in the sternum and reported a feeling of uncomfortable pressure within the chest. Adolescent and older patients developed moderate subcutaneous hematoma, which disappeared within a few hours.
Temporary side effects like transient paresthesia of the upper extremities during the application and/or mild dorsalgia were reported by some patients. These symptoms disappeared when lower atmospheric pressure was used during application. Analgesic medication was not necessary in any patient. The application of the vacuum bell in children aged from 3 to 10 years was supervised by the parents. In this age group, no relevant side effects were reported.

While starting with the application, patients presented with a PE with depth from 2 to $5 \mathrm{~cm}$. In 105 patients (79\%), after 3 months of treatment, an elevation of more than $1 \mathrm{~cm}$ was documented. In 18 patients $(13.5 \%)$, the sternum was lifted to a normal level after 18 months (Figs. 2 and 3). The longest follow-up after discontinuation is 5 years, and the success until today is permanent and still visible (Fig. 3). In three patients with asymmetric PE, the depth of PE has decreased after 9 months, but the asymmetry is still visible (Fig. 4). Three patients were dissatisfied with the postoperative result ( 2 patients after MIRPE and 1 patient after Ravitch procedure) and started treatment with the vacuum bell. Thirteen patients stopped the application after 19.9 months in average due to an unsatisfactory result (4 patients) and decreasing motivation (9 patients). Twelve patients underwent MIRPE. At followup, all patients were satisfied and expressed their motivation to continue the application, if necessary.

\section{Discussion}

The vacuum method was used as early as 1910 by Lange [19]. The vacuum bell used in our patients group was developed by an engineer, who himself suffered from PE. We use this method since 2003. Long-term evidence of persistent effects of the treatment modality for more than 5 years is not yet available. However, initial results proved dramatic [20,21], and the acceptance and compliance of
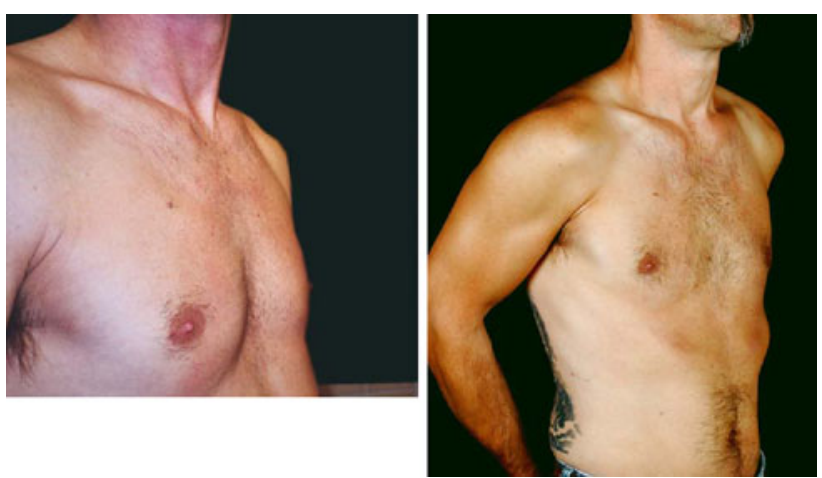

Fig. 2 Forty-five-year-old patient, before (left: depth of $\mathrm{PE}=$ $2.5 \mathrm{~cm}$ ) vacuum bell therapy and after 12 months (right: depth of $\mathrm{PE}=0.5 \mathrm{~cm})$ 


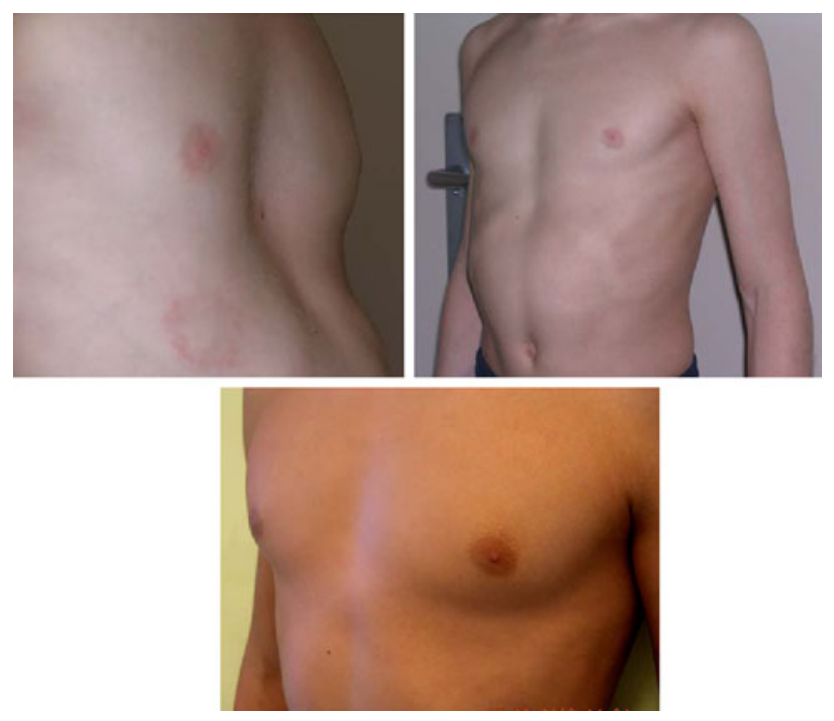

Fig. 3 Nine-year-old boy, before (left: depth of $\mathrm{PE}=2.8 \mathrm{~cm}$ ) vacuum bell therapy, after 10 months (right: depth of $P E=1.6 \mathrm{~cm}$ ) and 36 months after therapy (below)
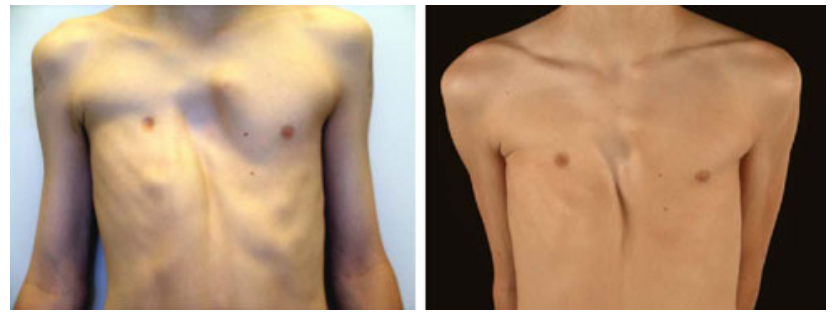

Fig. 4 Ten-year-old boy with asymmetric PE, before (left) vacuum bell therapy, and after 12 months (right)

patients seem to be good. Information on such new therapeutic modalities circulates not only among surgeons and pediatricians, but also rapidly among patients. In particular patients, who refused operative treatment by previously available procedures, now appear at the outpatient clinic and request to be considered for this method. From 1999 onwards, an increasing number of patients presented with $\mathrm{PE}$ at our department and asked to undergo the MIRPE procedure. In many cases of $\mathrm{PE}$, the degree of pectus deformity does not immediately warrant surgery, yet patients may benefit from some type of nonsurgical treatment. Other patients are disinclined to undergo surgery due to possible complications after surgery, because of the pain associated with postoperative recovery and the risk of imperfect results. Thus, the introduction of the vacuum bell for conservative treatment of $\mathrm{PE}$ has generated much interest among patients with PE.

The success of a therapeutic procedure not only requires a good technique, but also depends on an appropriate indication. In our study patients who presented with symmetric and mild PE seemed to show a more successful outcome than those with asymmetric and deep PE. All patients except 13 were satisfied with the use of the vacuum bell, although objectively assessed improvement of PE varied among the individuals. All our patients were recommended to carry on undertaking sports and physiotherapy, so that the accompanying improvement of body control was an important factor in outcome. The participation of patients themselves in the "active" treatment of PE clearly increases motivation to maintain therapy. As demonstrated in the CT scan, the force of the vacuum bell is strong enough to deform the chest within minutes [22]. Therefore, especially in children younger than 10 years of age, the application of the vacuum bell has to be performed carefully and should be supervised by an adult.

While creating the vacuum, the elevation of the sternum is obvious and persists for a distinct period of time. Therefore, the vacuum cup may also be useful in reducing the risk of injury to the heart during the MIRPE procedure. As described previously [20,21], we use the vacuum bell since 2006 routinely during the MIRPE procedure. In addition, the vacuum bell may be useful in a way of "pretreatment" to surgery.

To estimate the "objective" success of this treatment modality is very difficult. The definition of success may vary considerably between individuals. Depth of PE, symmetric versus asymmetric deformity, as well as patients' age and sex represent important variables. Various scales and measurement methods including X-rays and computed tomography have been used to quantify the degree of deformity. Our method of assessment of depth of PE is not exact enough, especially regarding the age specific differences. New methods for non-invasive assessment of chest wall growth may provide more detailed, objective information concerning the severity of PE [23]. Long-term results including 10 years and more are still missing. Further studies are necessary to elucidate these facts.

In conclusion, the vacuum bell may allow some patients with PE to avoid surgery. Especially, patients with symmetric and mild PE may benefit from this procedure. The application is easy, and we noticed a good acceptance by both pediatric and adult patients. However, the time of follow-up in our series is only 5 years, and further follow-up studies are necessary to evaluate the effectiveness of this therapeutic tool. Additionally, the intraoperative use of the vacuum bell during the MIRPE may facilitate the introduction of the pectus bar. In any case, the method seems to be a valuable adjunct therapy in the treatment of PE.

\section{References}

1. Molik KA, Engum SA, Rescorla FJ, West KW, Scherer LR, Grosfeld JL (2001) Pectus excavatum repair: experience with standard and minimal invasive techniques. J Pediatr Surg $36: 324-328$ 
2. Ravitch MM (1949) The operative treatment of pectus excavatum. Ann Surg 129:429-444

3. Nuss D, Kelly RE, Croitoru DP, Katz ME (1998) A 10-year review of a minimally invasive technique for the correction of pectus excavatum. J Pediatr Surg 33:545-552

4. Croitoru DP, Kelly RE, Goretsky MJ, Lawson ML, Swoveland B, Nuss D (2002) Experience and modification update for the minimally invasive Nuss technique for pectus excavatum repair in 303 patients. J Pediatr Surg 37:437-445

5. Hosie S, Sitkiewicz T, Petersen C, Göbel P, Schaarschmidt K, Till $\mathrm{H}$ et al (2002) Minimally invasive repair of pectus excavatum-the Nuss procedure. A European multicentre experience. Eur J Pediatr Surg 12:235-238

6. Nuss D, Croitoru DP, Kelly RE, Goretsky MJ, Nuss KJ, Gustin TS (2002) Review and discussion of the complications of minimally invasive pectus excavatum repair. Eur J Pediatr Surg 12:230-234

7. Haecker F-M, Bielek J, von Schweinitz D (2003) Minimally invasive repair of pectus excavatum (MIRPE): the Basel experience. Swiss Surg 9:289-295

8. Park HJ, Lee SY, Lee CS (2004) Complications associated with the Nuss procedure: analysis of risk factors and suggested measures for prevention of complications. J Pediatr Surg 39:391-395

9. Dzielicki J, Korlacki W, Janicka I, Dzielicka E (2006) Difficulties and limitations in minimally invasive repair of pectus excavatum-6 years experiences with Nuss technique. Eur J CardioThorac 30:801-804

10. Shin S, Goretsky MJ, Kelly RE, Gustin T, Nuss D (2007) Infectious complications after the Nuss repair in a series of 863 patients. J Pediatr Surg 42:87-92

11. Berberich T, Haecker F-M, Kehrer B, Erb T, Günthard J, Hammer J, Jenny P (2004) Postcardiotomy syndrome after minimally invasive repair of pectus excavatum. J Pediatr Surg 39:e1-e3

12. Van Renterghem KM, von Bismarck S, Bax NMA, Fleer A, Hoellwarth M (2005) Should an infected Nuss bar be removed? J Pediatr Surg 40:670-673
13. Barakat MJ, Morgan JA (2004) Haemopericardium causing cardiac tamponade: a late complication of pectus excavatum repair. Heart 90:e22-e23

14. Barsness K, Bruny J, Janik JS, Partrick DA (2005) Delayed nearfatal hemorrhage after Nuss bar displacement. J Pediatr Surg 40:E5-E6

15. Hoel TN, Rein KA, Svennevig JL (2006) A life-threatening complication of the Nuss-procedure for pectus excavatum. Ann Thorac Surg 81:370-372

16. Adam LA, Lawrence JL, Meehan JJ (2008) Erosion of the Nuss bar into the internal mammary artery 4 months after minimally invasive repair of pectus excavatum. J Pediatr Surg 43:394-397

17. Gips H, Zaitsev K, Hiss J (2008) Cardiac perforation by a pectus bar after surgical correction of pectus excavatum: case report and review of the literature. Pediatr Surg Int 24:617-620

18. Haecker F-M, Berberich T, Mayr J, Gambazzi F (2009) Nearfatal bleeding after transmyocardial ventricle lesion during removal of the pectus bar after the Nuss procedure. J Thorac Cardiovasc Surg 138 (5):1240-1. Epub 2008 Sep 19

19. Lange F (1910) Thoraxdeformitäten. In: Pfaundler M, Schlossmann A (eds) Handbuch der Kinderheilkunde, Vol V. Chirurgie und Orthopädie im Kindesalter. FCW Vogel, Leipzig, p 157

20. Haecker F-M, Mayr J (2006) The vacuum bell for treatment of pectus excavatum: an alternative to surgical correction? Eur J Cardio-Thorac 29:557-561

21. Haecker F-M (2009) Conservative treatment of pectus excavatum with the vacuum bell by E. Klobe: an alternative to the metal holder? Orthop praxis 45:183-189

22. Schier F, Bahr M, Klobe E (2005) The vacuum chest wall lifter: an innovative, nonsurgical addition to the management of pectus excavatum. J Pediatr Surg 40:496-500

23. Chang PY, Chang CH, Lai JY, Chen JC, Perng DB, Zeng Q (2010) A method for the non-invasive assessment of chest wall growth in pectus excavatum patients. Eur J Pediatr Surg 20:82-84 